\title{
The efficacy and safety of autologous conditioned serum (ACS) injections compared with betamethasone and placebo injections in the treatment of chronic shoulder joint pain due to supraspinatus tendinopathy: a prospective, randomized, double-blind, controlled study
}

\author{
Nemanja Damjanovin, Branko Barać1, Jelena Čolić1, Vladan Stevanović², Ana Zeković1, \\ Goran Tulić 3
}

${ }^{1}$ Institute of Rheumatology, ${ }^{2}$ Institute for Orthopedic Surgery "Banjica", ${ }^{3}$ Clinic for Orthopedic Surgery and Traumatology, Clinical Center of Serbia, University of Belgrade School of Medicine, Belgrade, Serbia

\begin{abstract}
Aims: Autologous conditioned serum (ACS; marketed as Orthokine ${ }^{\circledR}$ ) is an autologous blood product that has previously shown efficacy in treatment of joint osteoarthritis, spinal radiculopathy, tendon and muscle injuries in randomized controlled trials. In this 24-week, randomized, double-blind study, we compared the efficacy and safety of ACS with glucocorticoid (betamethasone) injections in chronic supraspinatus tendinopathy patients. Material and methods: Thirty-two patients with chronic supraspinatus tendinopathy were enrolled in the study. The ACS group received four ACS injections once weekly over four weeks and the glucocorticoid group received three betamethasone injections once weekly over three weeks with a placebo (saline) injection at week 4 into the enthesis and paratenon of the supraspinatus tendon. Study endpoints were pain intensity (VAS) and Constant Shoulder Score (CSS) assessed at weeks 0, 4 and 24. Results: Shoulder pain intensity improved after 4 weeks and significantly improved after 24 weeks in patients treated with ACS compared with those treated with glucocorticoids (pain intensity week 4: ACS=22.0, glucocorticoid=32.0; week 24: ACS=15.0, glucocorticoid=40.0). CSS improved to a similar extent in both groups after 4 weeks. After 24 weeks, ACS patients exhibited significantly greater CSS improvements than glucocorticoid patients. Adverse events $(n=8)$ were reported in betamethasone patients. Conclusions: Compared with betamethasone, ACS therapy improved joint function and reduced shoulder pain more effectively after 4 weeks of treatment; these improvements were sustained to week 24 . Combined with its favorable safety profile, ACS appears to be a more effective treatment than glucocorticoids and could enhance the quality of life in patients with chronic rotator cuff tendinopathy.

Keywords: autologous conditioned serum; betamethasone; chronic supraspinatus tendinopathy
\end{abstract}

\section{Introduction}

The rotator cuff consists of muscles and tendons that surround the top of the humerus providing support and a wide range of motion. Tendinopathy of the rotator cuff is the most common cause of shoulder pain in adults [1]

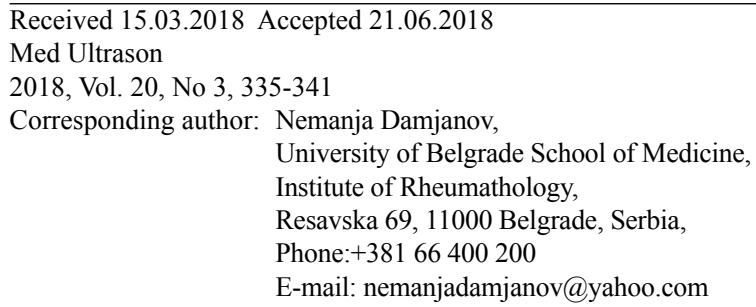

and can lead to a significant reduction in quality of life. Common symptoms include stiffness, dyskinesis, pain and weakness experienced during external rotation and elevation [2] which can cause considerable discomfort during simple daily tasks.

Subacromial corticosteroid injections are a common treatment for patients with rotator cuff tendinopathy, although some controversy remains as to how effective they are in the long term. Previous studies have reported small, short-term improvements with corticosteroid treatment of shoulder impingement [3,4]. However, other trials have demonstrated a lack of long-term efficacy for corticosteroid injections [5,6]. A systematic review investigating the efficacy of corticosteroid injections in the 
treatment of rotator cuff pathologies found little or no evidence to support the use of this treatment modality [6].

Autologous conditioned serum (ACS; marketed as Orthokine $\left.{ }^{\circledR}\right)$ is an autologous blood product used for the treatment of joint osteoarthritis, spinal radiculopathy, tendon and muscle injuries [7]. ACS has also shown promising results in animal studies. In a placebo-controlled study, horses with naturally occurring tendinopathies were given a single injection of ACS and in the ACS-treated limbs, the lameness decreased significantly up to day 10 of the treatment [8]. Similarly, in a study evaluating the effects of ACS on rats with sutured Achilles tendons, tendons exposed to ACS were thicker, had more type I collagen and an accelerated recovery of tendon stiffness [9].

ACS is derived solely from patient's blood, and, as it is cell-free, differs fundamentally from the alternative autologous blood therapy, platelet-rich plasma (PRP) [10]. ACS is produced by incubation of venous whole blood at physiological temperature (approximately $37^{\circ} \mathrm{C}$ ). This induces production of anti-inflammatory cytokines [7], including interleukin-1 receptor antagonist (IL-1-Ra), which is an important mediator of inflammation and tissue destruction in musculoskeletal conditions [11]. The cell-free supernatant, which contains these cytokines, is then extracted from the coagulated blood using centrifugation and re-injected into the affected tissue through a sterile filter. ACS has shown efficacy for treatment of knee and hip osteoarthritis (OA) in randomized controlled trials [12-14], as well as lumbar radicular compression [15] and muscular injuries [16].

Due to the avascular nature of tendons, their regenerative ability is limited [17]. There is some clinical evidence to suggest that by re-vascularizing the pathological area, recovery of the tendon can occur more readily [18]. Injection of orthobiologics, such as ACS and PRP, may help to achieve this process, and these treatments have subsequently been shown to aid tendon repair $[8,9,19$ 23]. Growth factors and cytokines, thought to stimulate the healing of soft tissue, are released upon platelet activation in situ after PRP injection. In a recent study comparing the efficacy of PRP and cortisone injections, PRP was found to be more effective after 12 weeks of treatment [24], although no statistical differences between the two treatments were observed after 6 months. In contrast, steroid injections were found to be more effective than PRP injections in the short (6 weeks) and long-term (6 months) treatment of subacromial impingement syndrome in a study of 60 patients [25].

Although animal studies have shown ACS to be effective in the treatment of tendinopathies, no current data are available detailing the effect of ACS tendinopathy treatment in humans. This is the first study to investigate the clinical application of ACS in patients with shoulder tendinopathy, a condition which is associated with chronic pain. Here, we compared the efficacy and safety of ACS with corticosteroid (betamethasone) injections for treatment of supraspinatus tendinopathy.

\section{Material and methods}

\section{Study design}

This prospective, randomized, double-blind, controlled study was conducted at the Institute of Rheumatology of Belgrade University over a 24-week period. At week 0, patients were randomized 1:1; the ACS group received four ACS injections once weekly over four weeks and the glucocorticoid group received three betamethasone injections once weekly over three weeks with a placebo (saline) injection at week 4 into the enthesis and paratenon of the supraspinatus tendon (fig 1).

\section{Patients}

To participate in the study, patients with supraspinatus tendinopathy were required to be $\geq 18$ years old. The main inclusion criterion for the study was a shoulder pain visual analog score (VAS) of $\geq 50 \mathrm{~mm}$, without any history of previous trauma. Additionally, all patients must have experienced shoulder pain for $\geq 6$ weeks, and not have received any treatment in the 2 weeks prior to study commencement, regardless of previous analgesic and physical therapy. In cases of acute shoulder pain, patients were permitted to take $500 \mathrm{mg}$ paracetamol every 4 hours when required. Patients were excluded if they had received any local glucocorticoid injections in the shoulder $\geq 24$ weeks before study commencement. Patients

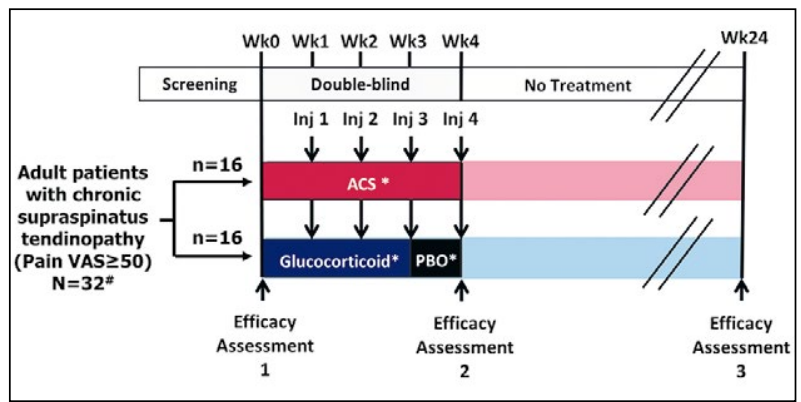

Fig 1. Study design. * One injection per week; \# Number of patients enrolled in the study. ACS: autologous conditioned serum; Inj: injection; PBO: placebo; Wk: week. Patients randomized to the ACS group received $4 \times 2 \mathrm{ml}$ serum injections (1 per week). Patients randomized to the glucocorticoid group received $3 \times 7 \mathrm{mg}$ injections ( 1 per week) betamethasone followed by 1 injection of saline (PBO) at week 4 . No treatment was administered after week 4 and patients completed a follow-up visit at week 24 
with contraindications for glucocorticoid treatment (diabetes mellitus, arterial hypertension, peptic ulcer, history of gastrointestinal bleeding) were also excluded from the study. Other exclusion criteria included inflammatory, rheumatic diseases, autoimmune, metabolic diseases and severe forms of acute or chronic diseases of other organ systems (infections and malignancies included).

All participants that fulfilled the inclusion criteria listed above received a diagnostic ultrasound of the shoulder to rule out any pathology that would exclude them from participation. Scanning was conducted at an appropriate temperature with the shoulder joint supported in a relaxed position. Only patients with ultrasonography indicative of supraspinatus tendinopathy were included, with or without signs of calcifications, enthesophytes and cortical bone abnormalities (erosions and/or osteophytes). Patients with supraspinatus tendon tears (partial or complete) or impingement, as indicated by ultrasonography, were excluded from the study. Patients with any other conditions that had the potential to jeopardize the study objective or violate study protocol were also excluded.

The study was approved by the local health authority and all patients provided informed consent in line with the Helsinki Declaration.

\section{Study procedures and assessments}

Venous blood samples were taken from each patient using an EOT ${ }^{\circledR} \mathrm{II}$ syringe [26] and incubated for 7 hours at $37^{\circ} \mathrm{C}$. The samples were then centrifuged $(3000 \mathrm{x} \mathrm{g})$ for 10 minutes before the serum was extracted. Injection of the ACS serum ( $2 \mathrm{ml}$ per injection) or betamethasone/ placebo was administered under the control of sonography [27-31] (half of the dose into the entheses and the other half into the paratenon of the supraspinatus tendon; fig 2). This was conducted by one sonographer and patients were blinded to which treatment they received. An independent rheumatologist, who was also blinded to treatment, examined patients at each visit.

In order to assess the efficacy of ACS treatment compared with betamethasone/placebo, shoulder pain and joint function were measured at weeks 0,4 and 24 . Shoulder pain was assessed using a visual analog scale (VAS), where a score of 0 indicated no pain, and a score of 100 indicated the most severe pain. Shoulder joint function was measured using the Constant Shoulder Score (CSS), which utilizes a $0-100 \mathrm{~mm}$ scale; a score of $<11$ was classed as Excellent, 11-20 is Good, 21-30 is Fair and $>30$ is Poor [32].

The safety profiles of ACS and betamethasone/placebo over 24 weeks was investigated through the analysis of adverse events (AEs) at each study visit. Safety assessments were conducted using the Safety Set, which

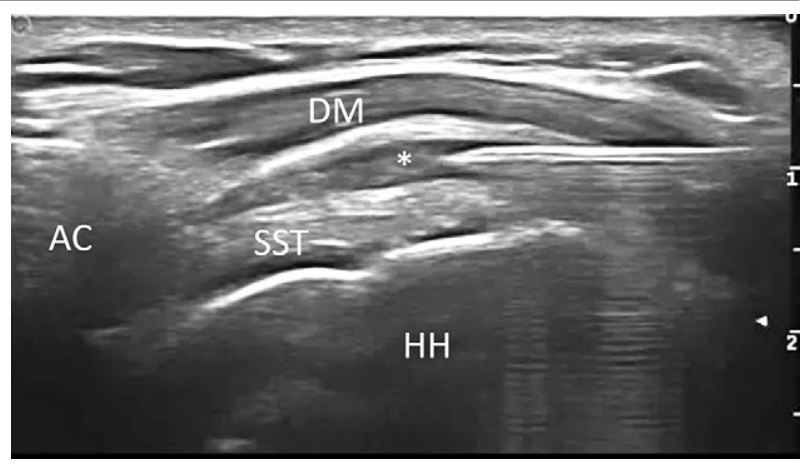

Fig 2. Ultrasound image showing injection of ACS into the supraspinatus tendon. *ACS ( $2 \mathrm{ml}$ per injection) was injected into the paratenon (fibrous sheath of the supraspinatus tendon); AC: acromion; DM: deltoid muscle; HH: humeral head; SST: supraspinatus tendon.

consisted of all patients who received $\geq 1$ dose of ACS or betamethasone. All AEs are reported here.

\section{Statistical analysis}

In this study, the data of continuous variables were expressed as mean \pm standard deviation (SD) or median (minimum-maximum), whereas categorical variables were presented using frequency counts (n, \%). Kolmogorov-Smirnov and Shapiro-Wilk tests were used to analyze distribution, as well as graphical methods.

Differences in investigated parameters were assessed using the Student's T test for continuous data with normal distributions. For continuous data with non-normal distribution variables, Mann-Whitney rank sum tests and Friedman and Wilcoxon signed rank tests were carried out. Spearman's method was used to analyze correlation. P-values of 0.05 or below were considered to be statistically significant. Statistical analyses were performed using the SPSS 17.0 software (SPSS Inc., Chicago, IL, USA).

\section{Results}

\section{Patient disposition and baseline characteristics}

A group of 32 patients (21 women and 11 men) was enrolled in the study: 16 patients were randomized to ACS and 16 patients to glucocorticoid and placebo. A total of 8 women and 8 men were assigned to the ACS group, whilst 13 women and 3 men were assigned to the glucocorticoid group. Full baseline demographics are given in Table I. There was no statistically significant difference in age and gender distribution between patients treated with ACS and those treated with glucocorticoids (betamethasone) and placebo ( $p>0.05$ ). During the study, one male patient from the ACS group withdrew informed consent before treatment and was not included in the fi- 
Table I. Baseline demographics and disease characteristics

\begin{tabular}{lll}
\hline Characteristic & $\begin{array}{l}\text { Glucocorticoid } \\
\mathbf{n = 1 6}\end{array}$ & $\begin{array}{l}\text { ACS } \\
\mathbf{n}=\mathbf{1 5}\end{array}$ \\
\hline Mean age, years (SD) & $64.87(11.8)$ & $59.00(15.1)$ \\
Female, n (\%) & $13(81.0)$ & $8(53.0)$ \\
VAS 0-100 mm, median & $65(50,100)$ & $70(50,90)$ \\
(min,max) & & \\
CSS, n (\%) & $14(87.5)$ & $13(86.7)$ \\
$\quad$ Poor & $2(12.5)$ & 0 \\
$\quad$ Fair & 0 & 0 \\
$\quad$ Good & 0 & $2(13.3)$ \\
$\quad$ Excellent &
\end{tabular}

Full analysis set. n: number of patients; CSS: Constant shoulder score; VAS: visual analog scale.

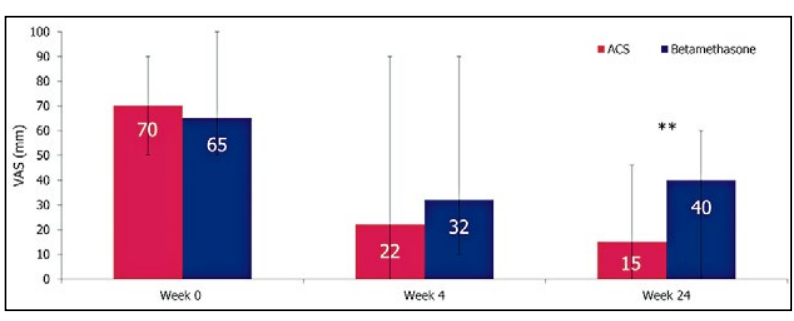

Fig 3. Shoulder pain (VAS) data at weeks 0,4 and 24. ** Highly significant: $\mathrm{p}=0.002$. VAS: visual analog scale for pain. Median values depicted. Error bars denote minimum and maximum values.

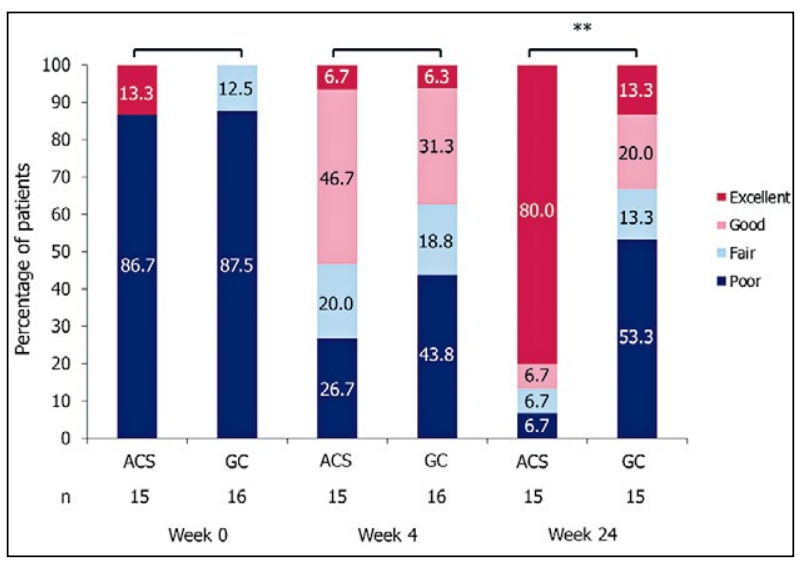

Fig 4. Shoulder function (CSS) data at weeks 0,4 and 24 of ACS and glucocorticoid-treated patients. $* *$ Highly significant: $\mathrm{p}<0.002$. Full analysis set. CSS: Constant shoulder score. GC: glucocorticoid. CSS scores: $<11$ is Excellent, 11-20 is Good, 21-30 is Fair and $>30$ is Poor.

nal analysis. One patient in the glucocorticoid and placebo group was lost to follow up at week 24.

\section{Efficacy data}

At baseline, the ACS and glucocorticoid/placebo groups exhibited similar levels of pain intensity (fig 3;
Table II. Summary of adverse events.

\begin{tabular}{lll}
\hline & $\begin{array}{l}\text { Glucocorticoid } \\
\mathbf{n = 1 6}\end{array}$ & $\begin{array}{l}\text { ACS } \\
\mathbf{n = 1 5}\end{array}$ \\
\hline Any AE (n) & 8 & 0 \\
Arterial hypertension & 2 & 0 \\
Facial erythema & 2 & 0 \\
Facies lunata & 1 & 0 \\
Headache & 3 & 0 \\
\hline
\end{tabular}

n: number of patients; AE: adverse event.

$\mathrm{p}=0.896)$. Comparison between the ACS and glucocorticoid groups after 4 weeks demonstrated no significant differences in pain intensity reduction between treatment options ( $\mathrm{p}=0.142$ ).

The ACS group displayed significantly lower median pain intensity scores at week 4 (22.0), which improved to week 24 (15.0), compared with baseline values $(\mathrm{p}=0.001$ for both).

Similarly, the glucocorticoid/placebo group exhibited a significantly lower median intensity score than baseline at week 4 (32.0; $\mathrm{p}=0.001)$, but with an increase in VAS score to week 24 (40.0; $\mathrm{p}=0.850)$. After 24 weeks of follow up, the ACS group exhibited a significantly better reduction in pain compared to the glucocorticoid/placebo group $(\mathrm{p}=0.002)$.

Similar levels of shoulder joint function were exhibited by the ACS and glucocorticoid/placebo groups at baseline (fig 4; $p=0.235$; Fisher's Exact Test $\chi^{2}=3,265$ ).

After 4 weeks of treatment, a significant improvement in shoulder function (CSS) was observed in patients treated with ACS injections $(\mathrm{p}=0.028$, Friedman and Wilcoxon signed ranks $\left.\chi^{2}=20,440\right)$. Similarly, patients in the glucocorticoid/placebo group showed significant improvement to week $4(\mathrm{p}=0.014)$. When the two treatment options were compared at week 4, CSS was similarly improved $(\mathrm{p}=0.779$; Fisher's Exact Test $\left.\chi^{2}=1,432\right)$.

At week 24, the ACS group exhibited significantly greater improvements in CSS compared with the glucocorticoid/placebo group ( $\mathrm{p}=0.001$, Fisher's Exact Test $\chi^{2}=14,059$ ). Contrastingly, CSS results in the glucocorticoid/placebo group were less improved. Despite this, significant improvements in the CSS of the glucocorticoid/placebo group were observed at week 24 in comparison with week $0(\mathrm{p}=0.023)$. However, deterioration in function, measured by CSS, was observed in the glucocorticoid/placebo group between week 4 and week 24 $(\mathrm{p}=0.539)$.

\section{Safety data}

Analysis of safety data revealed that 8 transient adverse events (AEs) were reported in three patients within 
the glucocorticoid/placebo group. These were listed as headache, arterial hypertension, facial erythema and facies lunata. No AEs were reported in the ACS group during the 24-week follow up period (Table II.). No serious AEs were reported in either treatment group.

\section{Discussions}

This study aimed to assess the efficacy and safety of ACS therapy in patients with supraspinatus tendinopathy associated with chronic pain and function lost during a 24-week period, compared with glucocorticoid/placebo treatment. It was concluded that ACS therapy is more efficacious when compared with betamethasone injections, although significant improvements were observed in both groups after 4 weeks of treatment. Intensity of pain in the shoulder (VAS) and shoulder function (CSS) improved further after 4 weeks and significantly more after 24 weeks of ACS treatment, compared with glucocorticoid/placebo treatment. Interestingly, 2 patients in the ACS group reported CSS results categorized as 'Excellent' at baseline, despite exhibiting pain scores $\geq 50 \mathrm{~mm}$. One possible explanation could be that, prior to suffering with supraspinatus tendinopathy, these two patients exhibited a higher than average range of shoulder motion. Onset of tendinopathy may, therefore, have reduced this range of motion, but not sufficiently to increase the CSS score above the 'Excellent' threshold.

The efficacy results of this trial are in agreement with previous studies, which observed a lack of longterm efficacy for corticosteroid treatment [6,33,34]. One trial $(\mathrm{n}=164)$ found that local corticosteroid injections in patients with lateral epicondylitis reduced pain more effectively than naproxen after 4 weeks. However, 12-month results suggested that the corticosteroid injections did not influence long-term outcomes [35]. A systematic review found that treatment with corticosteroids demonstrated statistically significant improvements in pain, global improvement and grip strength in patients suffering with lateral epicondylitis, compared with placebo [36]. However, no beneficial effects were reported in intermediate or long-term follow-up. In contrast, a recent study found that patients with subacromial impingement syndrome, who had been treated with a single steroid injection, exhibited considerable improvements in VAS and CSS scores after 6 months and showed significantly better improvements than patients treated with PRP [25].

The 8 AEs that were observed in our study were present in the betamethasone group, whereas none were reported in the ACS group; no serious AEs occurred in either study group. This suggests that ACS has a more favorable safety profile than corticosteroids and is in agreement with previous studies, which reported no AEs in ACS-treated patients $[14,16]$. In a prospective, observational trial investigating the effects of ACS therapy in knee osteoarthritis patients, no serious side effects were observed [37]. Similarly, in the German Orthokine Osteoarthritis Trial (GOAT) RCT, which compared ACS, hyaluronan and placebo in 376 knee osteoarthritis patients, no serious side effects were observed over 2 years [13].

It is evident from this investigation and previous studies $[33,38,39]$ that corticosteroid injections can significantly reduce pain in the short-term. However, such therapies should be considered with caution. Corticosteroid application can result in the weakening of the injected tendon, which in turn increases the likelihood of rupturing $[40,41]$. This issue, combined with the concerns associated with surgical intervention (high failure rates in patients $>65$ years of age [42], increased risk and longer hospital stays), highlights the importance of orthobiologics, such as ACS, in the future of tendinopathy treatment.

This study is not without limitations: only a small number of patients was analyzed and no placebo control group was used. In addition, if a patient experienced a reduction in pain, they may have been more likely to resume previous physical activities, which in turn could lead to further tissue damage. This factor could not be controlled in this study and could lead to misreporting of results, although the overall effect of this is likely to be small since pain reduction was reported in both groups.

In conclusion, injection of ACS in patients with chronic pain caused by supraspinatus tendinopathy has an excellent safety profile and exhibits larger improvements in shoulder pain and function than glucocorticoid injections over a 24-week period. These results suggest that ACS represents an effective and well-tolerated alternative to current therapies and has the potential to become a more established treatment option in both rotator cuff and other tendinopathies.

Acknowledgements: The authors acknowledge Tanju Kaptan, MD, Orthogen AG, Germany, for publication coordination and Eleanor Thurtle, MChem, and Julia Bárdos, PhD, Costello Medical Consulting, UK, for writing and editorial assistance. The development of this manuscript was funded by Orthogen AG.

Disclosures: $\mathrm{EOT}^{\circledR} \mathrm{II}$ syringes for ACS production were provided by Orthogen AG. All authors had no conflicts of interest to report. 


\section{References}

1. Van der Windt DA, Koes BW, de Jong BA, Bouter LM. Shoulder disorders in general practice: incidence, patient characteristics, and management. Ann Rheum Dis 1995;54:959-964.

2. Lewis JS. Rotator cuff tendinopathy. Br J Sports Med 2009;43:236-241.

3. Akgün K, Birtane M, Akarırmak Ü. Is local subacromial corticosteroid injection beneficial in subacromial impingement syndrome? Clin Rheumatol 2004;23:496-500.

4. Blair B, Rokito AS, Cuomo F, Jarolem K, Zuckerman JD. Efficacy of Injections of Corticosteroids for Subacromial Impingement Syndrome. J Bone Joint Surg 1996;78:1685-1689.

5. Gaujoux-Viala C, Dougados M, Gossec L. Efficacy and safety of steroid injections for shoulder and elbow tendonitis: a meta-analysis of randomised controlled trials. Ann Rheum Dis 2009;68:1843-1849.

6. Koester MC, Dunn WR, Kuhn JE, Spindler KP. The efficacy of subacromial corticosteroid injection in the treatment of rotator cuff disease: a systematic review. J Am Acad Orthop Surg 2007;15:3-11.

7. Wehling P, Moser C, Frisbie D, et al. Autologous Conditioned Serum in the Treatment of Orthopedic Diseases. Biodrugs 2007;21:323-332.

8. Geburek F, Lietzau M, Beineke A, Rohn K, Stadler PM. Effect of a single injection of autologous conditioned serum (ACS) on tendon healing in equine naturally occurring tendinopathies. Stem Cell Res Ther 2015;6:126.

9. Majewski M, Ochsner PE, Liu F, Fluckiger R, Evans $\mathrm{CH}$. Accelerated healing of the rat Achilles tendon in response to autologous conditioned serum. Am J Sports Med 2009;37:2117-2125.

10. Dhillon MS, Behera P, Patel S, Shetty V. Orthobiologics and platelet rich plasma. Indian J Orthop 2014;48:1-9.

11. Evans CH, Chevalier X, Wehling P. Autologous Conditioned Serum. Phys Med Rehabil Clin N Am 2016;4:893908.

12. Baselga García-Escudero J, Miguel Hernández Trillos P. Treatment of Osteoarthritis of the Knee with a Combination of Autologous Conditioned Serum and Physiotherapy: A Two-Year Observational Study. PLoS One 2016;10:e0145551.

13. Baltzer AW, Moser C, Jansen SA, Krauspe R. Autologous conditioned serum (Orthokine) is an effective treatment for knee osteoarthritis. Osteoarthritis Cartilage 2009;17:152160.

14. Baltzer AW, Ostapczuk MS, Stosch D, Seidel F, Granrath M. A New Treatment for Hip Osteoarthritis: Clinical Evidence for the Efficacy of Autologous Conditioned Serum. Orthop Rev (Pavia) 2013;5:59-64.

15. Becker C, Heidersdorf S, Drewlo S, de Rodriguez SZ, Kramer J, Willburger RE. Efficacy of epidural perineural injections with autologous conditioned serum for lumbar radicular compression: an investigator-initiated, prospective, double-blind, reference-controlled study. Spine (Phila Pa 1976) 2007;32:1803-1808.
16. Wright-Carpenter T, Opolon P, Appell HJ, Meijer H, Wehling P, Mir LM. Treatment of Muscle Injuries by Local Administration of Autologous Conditioned Serum: Animal Experiments Using a Muscle Contusion Model. Int J Sports Med 2004;25:582-587.

17. Codman EA. The Shoulder: Rupture of the Supraspinatus Tendon and Other Lesions in or about the Subacromial Bursa. R.E. Kreiger, 1934.

18. Bosch G, Moleman M, Barneveld A, van Weeren PR, van Schie HT. The effect of platelet-rich plasma on the neovascularization of surgically created equine superficial digital flexor tendon lesions. Scand J Med Sci Sports 2011;21:554561.

19. Wasterlain AS, Braun HJ, and Dragoo JL. Platelet-Rich Plasma as a Treatment for Patellar Tendinopathy: A Double-Blind Randomized Controlled Trial (SS-60). Arthroscopy 2012;28:e31-e32.

20. Guelfi M, Pantalone A, Vanni D, Abate M, Guelfi MG, Salini V. Long-term beneficial effects of platelet-rich plasma for non-insertional Achilles tendinopathy. Foot Ankle Surg 2015;21:178-181.

21. Cole BJ, Karas V, Hussey K, Pilz K, Fortier LA. Hyaluronic Acid Versus Platelet-Rich Plasma: A Prospective, Double-Blind Randomized Controlled Trial Comparing Clinical Outcomes and Effects on Intra-articular Biology for the Treatment of Knee Osteoarthritis. Am J Sports Med 2017;45:339-346.

22. Dai WL, Zhou AG, Zhang H, Zhang J. Efficacy of PlateletRich Plasma in the Treatment of Knee Osteoarthritis: A Meta-analysis of Randomized Controlled Trials. Arthroscopy 2017;33:659-670.e1.

23. Shen L, Yuan T, Chen S, Xie X, Zhang C. The temporal effect of platelet-rich plasma on pain and physical function in the treatment of knee osteoarthritis: systematic review and meta-analysis of randomized controlled trials. J Orthop Surg Res 2017;12:16.

24. von Wehren L, Blanke F, Todorov A, Heisterbach P, Sailer J, Majewski M. The effect of subacromial injections of autologous conditioned plasma versus cortisone for the treatment of symptomatic partial rotator cuff tears. Knee Surg Sports Traumatol Arthrosc 2016;24:3787-3792.

25. Say F, Gürler D, Bülbül M. Platelet-Rich Plasma Versus Steroid Injection for Subacromial Impingement Syndrome. J Orthop Surg (Hong Kong) 2016;24:62-66.

26. Orthokine. The Orthokine Serum: Preparation in Six Simple Steps. Orthogen. Accessed 08 July 2018. https://orthogen.com/orthokin/wp-content/uploads/sites/2/2016/07/EOTII_FlowChart_Vers03_EN.pdf.

27. Wu T, Song HX, Dong Y, Li JH. Ultrasound-guided versus blind subacromial\&subdeltoid bursa injection in adults with shoulder pain: A systematic review and meta-analysis. Semin Arthritis Rheum 2015;45:374-378.

28. Lee SW, Tiu T, Roberts J, Lee B, Bartels MN, OhPark M. Point-of-Care Ultrasonography Findings and Care Use Among Patients Undergoing UltrasoundGuided Shoulder Injections. Am J Phys Med Rehabil 2018;97:56-61. 
29. Chang KV, Wu WT, Han DS, Özçakar L. Static and Dynamic Shoulder Imaging to Predict Initial Effectiveness and Recurrence After Ultrasound-Guided Subacromial Corticosteroid Injections. Arch Phys Med Rehabil 2017;98:1984-1994.

30. Rastogi AK, Davis KW, Ross A, Rosas HG. Fundamentals of Joint Injection. AJR Am J Roentgenol 2016;207:484494.

31. Kianmehr N, Hasanzadeh A, Naderi F, Khajoei S, Haghighi A. A randomized blinded comparative study of clinical response to surface anatomy guided injection versus sonography guided injection of hyaloronic acid in patients with primary knee osteoarthritis. Int J Rheum Dis 2018;21:134139.

32. Constant CR, Murley AH. A clinical method of functional assessment of the shoulder. Clin Orthop Relat Res 1987;214:160-164.

33. Coombes BK, Bisset L, Vicenzino B. Efficacy and safety of corticosteroid injections and other injections for management of tendinopathy: a systematic review of randomised controlled trials. Lancet 2010;376:1751-1767.

34. Hart L. Corticosteroid and Other Injections in the Management of Tendinopathies: A Review. Clin J Sport Med 2011;21:540-541.

35. Hay EM, Paterson SM, Lewis M, Hosie G, Croft P. Pragmatic randomised controlled trial of local corticosteroid injection and naproxen for treatment of lateral epicondylitis of elbow in primary care. BMJ 1999;319:964-968.
36. Smidt N, Assendelft WJ, van der Windt DA, Hay EM, Buchbinder R, Bouter LM. Corticosteroid injections for lateral epicondylitis: a systematic review. Pain 2002;96:23-40.

37. Baltzer A, Drever R, Granrath M, Wehling P. Intra-articular treatment of osteoarthritis using autologous interleukine-1 receptor (IL-1RA) conditioned serum. Dtsch Z Sportmed 2003;54:209-211.

38. Zheng XQ, Li K, Wei YD, Tie HT, Yi XY, Huang W. Nonsteroidal Anti-Inflammatory Drugs Versus Corticosteroid for Treatment of Shoulder Pain: Systematic Review and Meta-Analysis. Arch Phys Med Rehabil 2014;95:18241831.

39. Petri M, Dobrow R, Neiman R, Whiting-O'Keefe Q, Seaman WE. Randomized, double-blind, placebo-controlled study of the treatment of the painful shoulder. Arthritis Rheum 1987;30:1040-1045.

40. Dean BJ, Franklin SL, Murphy RJ, Javaid MK, Carr AJ. Glucocorticoids induce specific ion-channel-mediated toxicity in human rotator cuff tendon: a mechanism underpinning the ultimately deleterious effect of steroid injection in tendinopathy? Br J Sports Med 2014;48:1620-1626.

41. Haraldsson BT, Langberg H, Aagaard P, et al. Corticosteroids Reduce the Tensile Strength of Isolated Collagen Fascicles. Am J Sports Med 2006;34:1992-1997.

42. Boileau P, Brassart N, Watkinson DJ, Carles M, Hatzidakis AM, Krishnan SG. Arthroscopic Repair of Full-Thickness Tears of the Supraspinatus: Does the Tendon Really Heal? J Bone Joint Surg 2005;87:1229-1240. 